\title{
MusE GAs FLOw and Wind (MEGAFLOW) VII: A NOEMA pilot program to probe molecular gas in galaxies with measured circumgalactic gas flows
}

\author{
Jonathan Freundlich, ${ }^{1,2,3 \star}$ Nicolas Bouché, ${ }^{4}$ Thierry Contini,${ }^{5}$ Emanuele Daddi, ${ }^{6}$ \\ Johannes Zabl, ${ }^{4}$ Ilane Schroetter, ${ }^{7}$ Leindert Boogaard, ${ }^{8}$ Johan Richard ${ }^{4}$ \\ ${ }^{1}$ School of Physics and Astronomy, Tel Aviv University, Tel Aviv 69978, Israel \\ ${ }^{2}$ Centre for Astrophysics and Planetary Science, Racah Institute of Physics, The Hebrew University, Jerusalem 91904, Israel \\ ${ }^{3}$ Université de Strasbourg, CNRS UMR 7550, Observatoire astronomique de Strasbourg, 67000 Strasbourg, France \\ ${ }^{4}$ Univ Lyon, Univ Lyon1, Ens de Lyon, CNRS, Centre de Recherche Astrophysique de Lyon UMR5574, F-69230 Saint-Genis-Laval, France \\ ${ }^{5}$ Institut de Recherche en Astrophysique et Planétologie (IRAP), Université de Toulouse, CNRS, UPS, F-31400 Toulouse, France \\ ${ }^{6}$ CEA, Irfu, DAp, AIM, Université Paris-Saclay, Université de Paris, CNRS, F-91191 Gif-sur-Yvette, France \\ ${ }^{7}$ GEPI, Observatoire de Paris, CNRS-UMR8111, PSL Research University, Univ. Paris Diderot, 5 place Jules Janssen, 92195 Meudon, France \\ ${ }^{8}$ Leiden Observatory, Leiden University, P.O. Box 9513, NL-2300 RA Leiden
}

Accepted XXX. Received YYY; in original form ZZZ

\begin{abstract}
We present a pilot program using IRAM's NOrthern Extended Millimeter Array (NOEMA) to probe the molecular gas reservoirs of six $z=0.6-1.1$ star-forming galaxies whose circumgalactic medium has been observed in absorption along quasar lines-of-sight as part of the MusE GAs FLOw and Wind (MEGAFLOW) survey and for which we have estimates of either the accretion or the outflow rate. This program is motivated by testing the quasi equilibrium model and the compaction scenario describing the evolution of galaxies along the main sequence of star formation, which imply tight relations between the gas content, the star formation activity, and the amount of gas flowing in and out. We report individual carbon monoxide $\mathrm{CO}(4-3), \mathrm{CO}(3-2)$ and dust continuum upper limits, as well as stacked $\mathrm{CO}$ detections over the whole sample and the three galaxies identified with outflows. The resulting molecular gas fractions and depletion times are compatible with published scaling relations established within a mass-selected sample, indicating that galaxies selected through their absorption follow similar relations on average. We further detect the dust continuum of three of the quasars and a strong emission line in one of them, which we identify as $\mathrm{CO}(4-3)$. Extending the sample to more galaxies and deeper observations will enable to quantify how the molecular gas fraction and depletion time depend on the inflow and ouflow rates.
\end{abstract}

Key words: galaxies:evolution - galaxies:haloes - intergalactic medium - quasars: absorption lines

\section{INTRODUCTION}

One of the main open questions in galaxy evolution is related to the processes responsible for the star formation regulation in galaxies. Observations have shown that, since at least $z \sim 4$, star-forming galaxies (SFG) follow a relatively tight and almost linear relation between their stellar mass $\left(M_{\star}\right)$ and star formation rate (SFR), the so-called star formation "main-sequence" (MS; Noeske et al. 2007; Elbaz et al. 2007, 2011; Daddi et al. 2007; Schiminovich et al. 2007; Rodighiero et al. 2010; Sargent et al. 2012; Whitaker et al.

^ E-mail: jonathan.freundlich@astro.unistra.fr
2012, 2014; Speagle et al. 2014; Renzini \& Peng 2015; Boogaard et al. 2018). This redshift-dependent and robust relation promotes an overall smooth and continuous mode for star formation in galaxies, which could be sustained by a continuous supply of gas from the cosmic web and minor mergers (Birnboim \& Dekel 2003; Kereš et al. 2005, 2009; Dekel \& Birnboim 2006; Ocvirk et al. 2008; Dekel et al. 2009; Genel et al. 2010) that provides large reservoirs of molecular gas to fuel star formation (Erb et al. 2006; Daddi et al. 2010; Tacconi et al. 2010, 2013, 2018; Sargent et al. 2014; Freundlich et al. 2019; Genzel et al. 2015). The rotating disc morphology of most galaxies that constitute this sequence (Förster Schreiber et al. 2006; Genzel et al. 2006, 2008; Stark et al. 2008; 
Daddi et al. 2010; Wuyts et al. 2011a) and the long star formation cycles inferred from the number of SFGs observed at $z=1-2$ (Daddi et al. 2005, 2007; Caputi et al. 2006) further argue in favour of such a smooth mode of star formation. Typical SFGs are thought to evolve along the MS in a slowly evolving gas-regulated quasi equilibrium between inflows, outflows, and star formation (Bouché et al. 2010; Davé et al. 2011, 2012; Feldmann 2013; Lilly et al. 2013; Dekel et al. 2013; Peng \& Maiolino 2014; Dekel \& Burkert 2014), until their star formation is quenched when they enter denser environment or reach a typical stellar mass of $\sim 10^{11} M_{\odot}$ (Conroy \& Wechsler 2009; Peng et al. 2010). SFGs then rapidly cease their star formation and drop below the MS to populate the "red sequence". Before this final quenching, SFGs may oscillate within the scatter of the MS through episodes of gas compaction, enhanced star formation above the MS line, gas depletion due to star formation, limited quenching below the MS line, and replenishment by external accretion as suggested by cosmological simulations (Dekel \& Burkert 2014; Zolotov et al. 2015; Tacchella et al. 2016a,b; Dekel et al. 2019, 2020b,a). Both the quasi equilibrium (or "bathtub") model and these oscillations around the MS line imply tight relations between the gas content of SFGs, the amount of gas flowing in and out, and the star formation activity. Under the quasi equilibrium model, mass conservation notably implies a relation between gas fraction and stellar mass, and one can in principle put indirect constraints on outflow rates from molecular gas observations as in Seko et al. (2016).

Observationally testing such relations is challenging, notably as signatures of gas flows are difficult to observe directly and as their location is highly uncertain when probed via standard galaxy emission or absorption lines. For example, outflow rates measured traditionally using blue-shifted absorption lines have order-of-magnitude uncertainties because the location of the absorbing gas is unknown (it can be located at $0.1,1$, or even 10 kpc away from the host galaxy). Absorption along serendipitous lines of sight of background quasars enables to significantly reduce these uncertainties by probing the circumgalactic medium (CGM) of SFGs around a given impact parameter (Bouché et al. 2012, 2013, 2016; Kacprzak et al. 2014; Muzahid et al. 2015; Schroetter et al. 2015). Despite the scarcity of galaxy-quasar pairs, the unprecedented field-of-view and sensitivity of the Multi Unit Spectroscopic Explorer (MUSE; Bacon et al. 2006, 2010) instrument together with the high-resolution Ultraviolet and Visual Echelle Spectrograph (UVES; Dekker et al. 2000) on the Very Large Telescope (VLT) have enabled to isolate a sample of about 100 SFGs at $z=0.5-1.4$ with a background quasar within $100 \mathrm{kpc}$ as part of the MusE GAs FLOw and Wind (MEGAFLOW) survey (Schroetter et al. 2016, 2019; Zabl et al. 2019, 2020). These galaxies were selected through their $\mathrm{Mg}$ II absorption on the lines-of-sight of 22 quasars from the Zhu \& Ménard (2013) catalog and constitute the largest sample of $\mathrm{Mg}_{\text {II }}$ absorber-galaxy pairs with spectroscopic and kinematic information to date. The azimuthal angle between the quasar location and the galaxy major axis follows a clear bimodality, indicating that strong $\mathrm{Mg}$ II absorption lies predominantly in outflow cones and extended disk-like structures (cf. also Bordoloi et al. 2011; Bouché et al. 2012; Kacprzak et al. 2012). This is further corroborated by cosmological simulations, which predict more substantial inflow (outflow) detections along the galaxy major (minor) axis (e.g., Péroux et al. 2020), and by the observed correlation between metallicity and azimuthal angle, gas located along the galaxy minor axis being on average more enriched than that located along the major axis (Wendt et al. 2020). Pairs with azimutal angle compatible with outflows were studied in Schroetter et al.
(2016), Schroetter et al. (2019) and Zabl et al. (2020) while pairs compatible with accretion around the disc plane were studied in Zabl et al. (2019). There has been no attempt so far to unveil the molecular gas content, i.e., the fuel reservoir for future star formation, in the SFGs caught in the act with outflowing or accreting gas by the MEGAFLOW survey.

In this paper, we present the NOEMA MEGAFLOW pilot program, a first attempt at probing molecular gas reservoirs in galaxies with gas flows drawn from the MEGAFLOW survey with IRAM's NOrthern Extended Millimeter Array (NOEMA; Schuster 2014). The primary objectives of this pilot program is to characterize the molecular gas content in a small but well-defined sample of galaxies with clear detections of either infalling or ouflowing gas, for which we have estimates of the gas accretion or ouflow rates, and to compare the resulting molecular gas fraction and depletion time with existing molecular gas measurements and scaling relations such as those of the PHIBSS and PHIBSS2 IRAM programs (Tacconi et al. 2010, 2013, 2018; Genzel et al. 2013, 2015; Freundlich et al. 2013, 2019). These programs provide an ideal comparison sample, as their targets were selected to uniformly sample the MS and its scatter in the $M_{\star}$-SFR plane above given $M_{\star}$ and SFR thresholds, and include about 120 molecular gas measurements in the MEGAFLOW redshift range $(z=0.5-1.4)$. In particular, since a major outflow can lead to a gas depletion situation, comparing the gas fraction and depletion time of the MEGAFLOW outflow sample with those of the reference sample can validate (or not) the gas cycling event: if the gas properties of the outflow sample follow the Tacconi et al. (2018) scaling relations, it would mean that outflow events do not have a strong impact on the galaxy gas content, contrarily to the prediction from simulations; if the gas properties have smaller gas content or depend on the location on the MS, it would validate the models. From an initial proposed sample of 11 galaxies, the NOEMA MEGAFLOW program eventually includes only 6 targets with no clear individual detections but whose stack can be compared to the Tacconi et al. (2018) molecular gas scaling relations. This pilot program enables to lay the ground for more ambitious programs that would test the quasi equilibrium and compaction models with simultaneous individual measurements of gas flows and gas content at intermediate redshifts. Section 2 presents the sample, the observations, and the methodology of the program; Section 3 the resulting upper limits, the stacking analysis, and the comparison with the scaling relations; Section 4 concludes and discusses the results. We further present in Appendix B the additional quasar continuum detections we obtained. Throughout this paper, we asssume a flat $\Lambda \mathrm{CDM}$ universe with $\Omega_{\mathrm{m}}=0.3, \Omega_{\Lambda}=0.7$, and $H_{0}=70 \mathrm{~km} \mathrm{~s}^{-1} \mathrm{Mpc}^{-1}$.

\section{SAMPLE SELECTION AND OBSERVATIONS}

\subsection{The MEGAFLOW survey}

The MEGAFLOW strategy consists in selecting quasar lines-ofsight with at least three $\mathrm{Mg}_{\text {II }} \lambda \lambda 2797,2803$ absorbers from the Zhu \& Ménard (2013) catalog based on the Sloan Digital Sky Survey (SDSS; Ross et al. 2012; Alam et al. 2015). These Mg II absorbers are further selected to have redshifts in the range $0.4-1.45$, such that the $\left[\mathrm{O}_{\mathrm{II}}\right] \lambda \lambda 3727,3729$ galaxy emission lines fall within the MUSE wavelength range (4800 - $9300 \AA$ ), and rest-frame equivalent widths larger than $>0.5-0.8 \AA$ in order to have impact parameters $b \lesssim 100 \mathrm{kpc}$ given the anticorrelation between the Mg II equivalent width and the impact parameter (Lanzetta \& Bowen 1990; 
Steidel 1995; Chen et al. 2010; Bordoloi et al. 2011; Kacprzak et al. 2011; Nielsen et al. 2013; Werk et al. 2013). The resulting sample includes 79 strong $\mathrm{Mg}$ II absorbers with $0.51<z<1.45$ and 38 isolated galaxy-absorber associations within 22 quasar fields.

As can be seen in the left panel of Fig. 1, the distribution of the azimuthal angle $(\alpha)$ between the apparent quasar location and the galaxy major axis is bimodal, indicating that strong $\mathrm{Mg}_{\mathrm{II}}$ absorption is preferentially found either along the minor axis or along the major axis of the galaxies (Bouché et al. 2012; Kacprzak et al. 2012; Zabl et al. 2019; Schroetter et al. 2019). Mg II absorptions along the minor axis $\left(|\alpha| \geq 55^{\circ}\right)$ are identified with outflows (Schroetter et al. 2019), since outflows are expelling baryons from the galaxy perpendicularly to the star-forming disk in the direction of least resistance; $\mathrm{Mg}$ II absorptions along the major axis $\left(|\alpha|<40^{\circ}\right)$ are identified with accretion around the disc plane (Zabl et al. 2019).

Redshifts and [O $\mathrm{OI}$ ] fluxes are measured using the 3D morphokinematics fitting tool Galpak3D (Bouché et al. 2015) on the MUSE data; stellar masses are estimated as in Schroetter et al. (2019) from the tight correlation between the stellar mass and the dynamical estimator $S_{05}=\sqrt{0.5 \times V_{\max }^{2}+\sigma^{2}}$, which combines the rotational velocity $V_{\max }$ and the galaxy velocity dispersion $\sigma$ derived by Galpak3D, using the relation from Alcorn et al. (2018); SFRs are determined from the MUSE [O II] fluxes using the Kewley et al. (2004) relation corrected for extinction using the Calzetti et al. (2000) law with the strength of the extinction estimated from the $M_{\star}-E(B-V)$ relation of Garn \& Best (2010) and assuming a Chabrier (2003) initial mass function (IMF). More details can be found in Zabl et al. (2019). Typical uncertainties on the stellar mass and the SFR are about 0.2 dex (e.g. Wuyts et al. 2011b; Whitaker et al. 2014; Roediger \& Courteau 2015). Mass accretion and outflowing rates as well as mass loading factors can be determined from the $\mathrm{Mg}$ II equivalent widths, the impact parameters, and the Mg II kinematics (cf. Bouché et al. 2012, 2013; Schroetter et al. 2015, 2016, 2019; Zabl et al. 2019).

The right panel of Fig. 1 shows the location of the MEGAFLOW galaxies with respect to the MS in terms of their SFR offset from the MS,

$\delta \mathrm{MS}=\mathrm{SFR} / \mathrm{SFR}\left(\mathrm{MS} ; z, M_{\star}\right)$

where SFR(MS; $z, M_{\star}$ ) is the analytical prescription for the center of the MS as a function of redshift and stellar mass proposed in the compilation by Speagle et al. (2014). All MEGAFLOW galaxies lie within the scatter of the MS, although galaxies above $\log \left(M_{\text {star }} / M_{\odot}\right) \sim 10$, which are dominated by wind cases, tend to be slightly below the Speagle et al. (2014) MS line. This may suggest that outflows above this mass may induce a star formation decrease. This potential relation between $\delta \mathrm{MS}$ and gas flows will be investigated further with the full survey (Bouché et al., in prep.).

While the average $\log \left(M_{\text {star }} / M_{\odot}\right)$ and $\delta \mathrm{MS}$ of the Tacconi et al. (2018) sample are respectively 10.7 and 0.39 , those of the MEGAFLOW sample are 9.8 and -0.16 and those of the sample observed with NOEMA 10.3 and -0.07: the MEGAFLOW sample and the NOEMA subsample have lower stellar masses and offsets from the MS than the Tacconi et al. (2018) sample on average.

\subsection{The pilot program sample}

For the NOEMA MEGAFLOW pilot program presented here, we selected a subset of SFGs from the MEGAFLOW sample with measured accretion and outflow events, mass flux estimates, stellar masses, and SFRs. The sources were selected to optimize the
NOEMA CO(4-3) or $\mathrm{CO}(3-2)$ observing times given their SFR, stellar masses, and redshifts, assuming the Tacconi et al. (2018) scaling relations to assess their expected line fluxes. As such, the sample is notably biased towards higher SFRs. As shown in Fig. 1, the current sub-sample comprises 6 SFGs equally divided between accretion and wind cases. Table 1 summarizes some of the properties of the targets, including their R.A., DEC. coordinates, redshifts, stellar masses, SFRs, [O II] line widths, and Mg II rest-frame equivalent width in quasar sightline. Each full galaxy ID includes the quasar identifier, the absorber redshift, the impact parameter in arc$\mathrm{sec}$, and the position angle between quasar and galaxy in degrees. In the following, we use the absorber redshift as short ID. The positions and redshifts of the quasars are indicated in Table B1.

\subsection{Observations and data reduction}

To probe the molecular gas content of the sample galaxies, we observe either the $\mathrm{CO}(3-2)$ or the $\mathrm{CO}(4-3)$ carbon monoxide emission lines $\left(v_{\text {rest }}=345.8\right.$ and $461.0 \mathrm{GHz}$, respectively), which fall in NOEMA's $1.3 \mathrm{~mm}$ band given the redshift range of the targets and are both located near the peak of the CO line SED for SFGs (e.g., Daddi et al. 2015). The six galaxies were observed at the NOEMA interferometer at the Plateau de Bure in France between March and June 2019, as part of the observational program W18CS (P.I.: N. Bouché, T. Contini). The $\mathrm{CO}(3-2)$ and $\mathrm{CO}(4-3)$ emission lines from the targets were observed at frequencies between 203 and $266 \mathrm{GHz}$, falling in the $3 \mathrm{~mm}$ band, in the extended D configuration with 10 antennas until beginning April 2019 and 9 afterwards. The beam size, the eventual on-source observing time for each target as well as the resulting RMS noise are indicated in Table 2. The weather conditions varied from good to mediocre, with system temperatures ranging between 100 and $500 \mathrm{~K}$ depending on atmospheric conditions, wind speeds between 1 and $13 \mathrm{~m} / \mathrm{s}$, and water vapor between 0.5 and $5 \mathrm{~mm}$. The absolute flux scale was derived from secondary flux calibrators ( $\mathrm{LkH} \alpha 101,0735+178$, 0923+392, MWC349, 0829+046, and 3C454.3), whose fluxes are regularly measured using Jupiter satellites or planets. The data were calibrated using the clic software of the IRAM gildas package, and further analysed and mapped using the gildas mapping and class sofwares.

\section{RESULTS}

\subsection{CO molecular gas}

With beam sizes (Table 2) at least 2-3 times larger than the average galaxy size on the MS at the redshift and stellar mass of the sources (van der Wel et al. 2014), our NOEMA observations are unresolved. For each observed galaxy, we extract the spectrum at the optical position by fitting the point spread function (PSF) in the $u v$ Fourier space using gildas mapping go uvfit tool. Fitting the unresolved sources through their $u v$ visibilities avoids the imaging and deconvolution steps, which involve assumptions to compensate for the sparse $u v$ coverage. The $\mathrm{CO}(3-2)$ and $\mathrm{CO}(4-3)$ spectra of the six galaxies of the sample are displayed in Fig. A1. We use the MUSE [O II] FWHM to evaluate the full width at tenth-maximum (FWTM) of the expected $\mathrm{CO}$ line assuming a Gaussian profile, i.e., FWTM $=$ FWHM $\times \sqrt{\log (10) / \log (2)}$, and evaluate both the signal and the noise within the FWTM - which is expected to contain 97\% of the flux. Indeed, since the targetted galaxies are typical MS SFGs, obscuration is not extreme and both $[\mathrm{O} \mathrm{II}]$ and $\mathrm{CO}$ should 

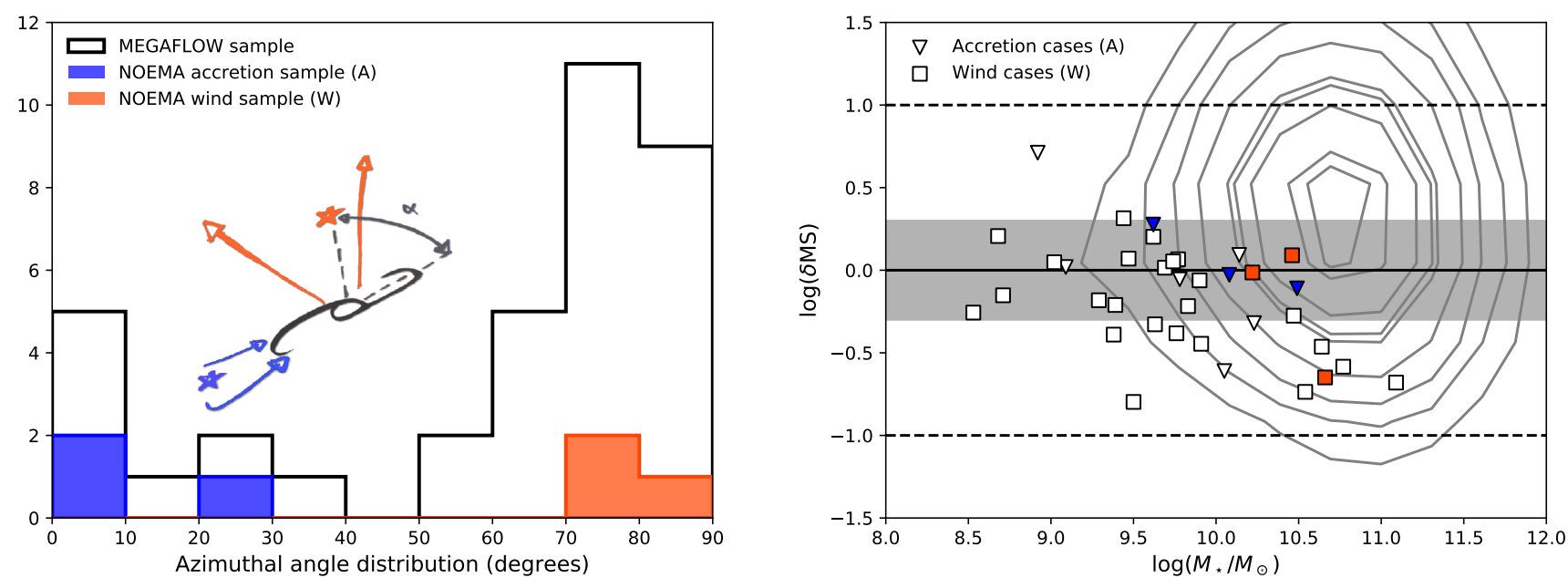

Figure 1. Left: Distribution of the azimuthal angle between the galaxy major axis and the quasar line-of-sight for the entire MEGAFLOW survey (plain black line) and for the sample observed with NOEMA (blue and red filled histograms). Accretion cases are represented in blue, wind cases in red. The blue (red) star in the overlaid drawing represents the position of the quasar in an accretion (wind) configuration. Right: Position of the MEGAFLOW galaxies with respect to the MS, where the offset from the MS $\left(\delta \mathrm{MS}=\mathrm{SFR} / \mathrm{SFR}\left(\mathrm{MS} ; z, M_{\star}\right)\right)$ is plotted as a function of the stellar mass $\left(M_{\star}\right)$ and the reference SFR(MS; $\left.z, M_{\star}\right)$ is that of Speagle et al. (2014). Empty triangles and squares are respectively the MEGAFLOW accretion and wind galaxies, those highlighted in blue and red correspond to the observed ones. The gray background contours indicate the Tacconi et al. (2018) sample, the plain horizontal line the MS ridge $(\delta M S=1)$, the shaded area its 0.3 dex scatter, and the dashed line \pm 1 dex from it.

Table 1. Parameters of the six galaxies observed as part of the NOEMA MEGAFLOW pilot program. (1) Galaxy ID, with the short ID highlighted in bold. (2) Type: A for an accretion case $\left(|\alpha|<40^{\circ}\right)$, W for a wind case $\left(|\alpha| \geq 55^{\circ}\right)$. (3)(4) Spatial coordinates. (5)(6)(7)(8)(9) Redshift, stellar mass, SFR, [O II] FWHM, and $\mathrm{Mg}$ II rest-frame equivalent width in quasar sightline (cf. Zabl et al. 2019; Schroetter et al. 2019).

\begin{tabular}{|c|c|c|c|c|c|c|c|c|}
\hline $\begin{array}{l}\text { ID } \\
(1)\end{array}$ & $\begin{array}{l}\text { Type } \\
(2)\end{array}$ & $\begin{array}{l}\text { R.A. } \\
\text { (3) }\end{array}$ & $\begin{array}{c}\text { DEC. } \\
(4)\end{array}$ & $\begin{array}{c}z \\
(5)\end{array}$ & $\begin{array}{c}\log \left(\mathrm{M}_{\mathrm{star}} / \mathrm{M}_{\odot}\right) \\
(6)\end{array}$ & $\begin{array}{c}\mathrm{SFR}\left[\mathrm{M}_{\odot} \mathrm{yr}^{-1}\right] \\
(7)\end{array}$ & $\begin{array}{c}\text { FWHM }[\mathrm{km} / \mathrm{s}] \\
(8)\end{array}$ & $\begin{array}{c}W_{\mathrm{r}}^{\lambda 2796}[\AA] \\
\quad(9)\end{array}$ \\
\hline gal_J0800p1849_0608_10_108 & A & 08:00:05.200 & $+18: 49: 32.550$ & 0.6082 & 9.62 & 4.99 & 180 & 0.8 \\
\hline gal_J1236p0725_0912_2_246 & A & $12: 36: 24.250$ & $+07: 25: 50.580$ & 0.9128 & 10.49 & 12.96 & 375 & 2.2 \\
\hline gal_J1039p0714_0949_6_324 & A & $10: 39: 36.420$ & $+07: 14: 32.370$ & 0.9494 & 10.08 & 8.78 & 235 & 1.2 \\
\hline gal_J0838p0257_1099_8_160 & $\mathrm{W}$ & $08: 38: 52.200$ & $+02: 56: 56.600$ & 1.1001 & 10.22 & 13.83 & 170 & 0.1 \\
\hline gal_J0937p0656_0702_6_209 & $\mathrm{W}$ & 09:37:49.400 & $+06: 56: 51.640$ & 0.7021 & 10.46 & 13.42 & 270 & 1.8 \\
\hline gal_J0015m0751_0731_5_3 & $\mathrm{W}$ & $00: 15: 35.190$ & $-07: 50: 57.890$ & 0.7313 & 10.66 & 3.49 & 217 & 2.1 \\
\hline
\end{tabular}

Table 2. CO observations. (1) Short ID, i.e., the identifier of the absorber redshift in the full galaxy ID quoted in Table 1. (2) Targetted CO transition. (3) Beam size. (4) Total on-source time. (5) RMS noise over the FWTM.

\begin{tabular}{|c|c|c|c|c|}
\hline $\begin{array}{l}\text { ID } \\
(1)\end{array}$ & $\begin{array}{l}\text { CO line } \\
\text { (2) }\end{array}$ & $\begin{array}{l}\text { beam } \\
(3)\end{array}$ & $\begin{array}{c}\mathrm{t}_{\mathrm{obs}}[\mathrm{h}] \\
(4)\end{array}$ & $\begin{array}{c}\sigma_{\text {FWTM }}[\mathrm{Jy} \mathrm{km} / \mathrm{s}] \\
(5)\end{array}$ \\
\hline 0608 & $3-2$ & $2.55^{\prime \prime} \times 2.15^{\prime \prime}$ & 4.1 & 0.11 \\
\hline 0912 & $4-3$ & $2.05^{\prime \prime} \times 1.24 "$ & 0.8 & 0.17 \\
\hline 0949 & $4-3$ & $3.09 " \times 1.51 "$ & 3.0 & 0.19 \\
\hline 1099 & $4-3$ & $2.39^{\prime \prime} \times 1.60 "$ & 0.9 & 0.17 \\
\hline 0702 & $3-2$ & $2.99 " \times 1.76 "$ & 1.4 & 0.15 \\
\hline 0731 & $4-3$ & $3.13 " \times 1.47 "$ & 3.4 & 0.24 \\
\hline
\end{tabular}

trace similar star-forming regions and velocity fields. Observational studies have also shown that $[\mathrm{O}$ II] and $\mathrm{CO}$ line widths were comparable (e.g., Freundlich et al. 2013, Puglisi et al. 2020, Nature Astronomy in press). The RMS noise indicated in Table 2 were obtained using gildas mapping go noise tool over velocity channels of width $\sim 50 \mathrm{~km} / \mathrm{s}$, rescaled to the [O II] FWTM. None of the galaxies is detected, although there may be hints of detection at low signal-to-noise ratio (SNR) for galaxies 0702 and 0731 . For these two galaxies, the velocity integrated line fluxes within the FWTM are respectively $0.280 \mathrm{Jy} \mathrm{km} / \mathrm{s}(\mathrm{SNR}=1.9)$ and $0.567 \mathrm{Jy} \mathrm{km} / \mathrm{s}$
(SNR = 2.4). Given the low SNR, we prefer to treat these measurements as non-detections. We nevertheless highlight that these fluxes and SNR estimates are obtained without any free parameters, since the positions and widths of the expected lines are set. We define $3 \sigma$ integrated line flux upper limits as $F_{\text {upper }}=3 \times \sigma_{\text {FWTM }}$, which correspond to the fluxes that should have been detected at SNR $>3$ with a 50\% probability (e.g., Masci 2011).

From any velocity integrated $\mathrm{CO}(J \rightarrow J-1)$ transition line flux $F_{\mathrm{CO}(J \rightarrow J-1)}$ (or upper limits $F_{\text {upper }}$ ), one can derive the intrinsic CO luminosity

$\left(\frac{L_{\mathrm{CO}(J \rightarrow J-1)}^{\prime}}{\mathrm{K} \mathrm{km} \mathrm{s}^{-1} \mathrm{pc}^{2}}\right)=\frac{3.25 \times 10^{7}}{1+z}\left(\frac{F_{\mathrm{CO}(J \rightarrow J-1)}}{\mathrm{Jy} \mathrm{km} \mathrm{s}^{-1}}\right)\left(\frac{v_{\mathrm{rest}}}{\mathrm{GHz}}\right)^{-2}\left(\frac{D_{\mathrm{L}}}{\mathrm{Mpc}}\right)^{2}$,

where $v_{\text {rest }}$ is the rest-frame frequency $(345.8 \mathrm{GHz}$ for $\mathrm{CO}(3-2)$, 461.041.8 GHz for $\mathrm{CO}(4-3))$, and $D_{\mathrm{L}}$ the luminosity distance (Solomon et al. 1997). Even if the CO molecule only represents a small fraction of the total molecular gas mass and if its lower rotational lines are almost always optically thick, this quantity can be used as a quantitative tracer of the molecular gas mass, estimated as

$M_{\mathrm{gas}}=\alpha_{\mathrm{CO}} L_{\mathrm{CO}(J \rightarrow J-1)}^{\prime} / r_{\mathrm{J} 1}$, 
Table 3. Quantities derived from the CO molecular gas measurements: intrinsic $\mathrm{CO}(1-0)$ luminosity, molecular gas mass, gas-to-stellar mass ratio, and depletion time. The table includes both the individual upper limits and the stacked values for all galaxies, for the accretion cases (A), and for the wind cases (W). Galaxies are referred to with their short ID (cf. Table 1).

\begin{tabular}{lllll}
\hline \hline ID & $L_{\mathrm{CO}(1-0)}^{\prime}\left[\mathrm{Kkms}^{-1} \mathrm{pc}^{2}\right]$ & $M_{\text {gas }}\left[M_{\odot}\right]$ & $\mu_{\text {gas }}$ & $t_{\text {depl }}[\mathrm{Gyr}]$ \\
\hline 0608 & $<1.310^{9}$ & $<7.510^{9}$ & $<1.80$ & $<1.5$ \\
0912 & $<3.410^{9}$ & $<1.5010^{10}$ & $<0.49$ & $<1.2$ \\
0949 & $<4.110^{9}$ & $<2.1910^{10}$ & $<1.82$ & $<2.5$ \\
\hline 1099 & $<4.910^{9}$ & $<2.5810^{10}$ & $<1.55$ & $<1.9$ \\
0702 & $<2.310^{9}$ & $<9.9610^{9}$ & $<0.35$ & $<0.7$ \\
0731 & $<3.110^{9}$ & $<1.2410^{10}$ & $<0.27$ & $<3.6$ \\
\hline stack & $1.410^{9}$ & $6.9610^{9}$ & 0.39 & 0.7 \\
A stack & $<1.610^{9}$ & $<8.2410^{9}$ & $<0.71$ & $<0.9$ \\
W stack & $2.110^{9}$ & $9.4210^{9}$ & 0.34 & 0.9 \\
\hline
\end{tabular}

where $\alpha_{\mathrm{CO}}$ is the $\mathrm{CO}(1-0)$ luminosity-to-molecular-gas-mass conversion factor and $r_{J 1}=L_{\mathrm{CO}(J \rightarrow J-1)}^{\prime} / L_{C O(1-0)}^{\prime}$ the corresponding line ratio. Since $\mathrm{CO}$ emission in the $z=0.6-1.1$ galaxies studied in this paper is likely to originate from virialized giant molecular clouds with mean densities of the same order of magnitude as their lowerredshift counterparts and similar dust temperatures, the conversion factor should be relatively close to the Galactic conversion factor $\alpha_{G}=4.36 \pm 0.9 \mathrm{M}_{\odot} /\left(\mathrm{K} \mathrm{km} \mathrm{s}^{-1} \mathrm{pc}^{2}\right)$. To estimate molecular gas mass upper limits from the $\mathrm{CO}(3-2)$ and $\mathrm{CO}(4-3)$ upper limits, we follow the PHIBSS2 methodology (Genzel et al. 2015; Tacconi et al. 2018; Freundlich et al. 2019). Namely, we include a metallicity dependence of the conversion factor, taken as the geometric mean of the recipes by Bolatto et al. (2013) and Genzel et al. (2012),

$\alpha_{\mathrm{CO}}=\alpha_{G} \sqrt{0.67 \times \exp \left(0.36 \times 10^{8.67-\log Z}\right) \times 10^{-1.27 \times(\log Z-8.67)}}$,

where $\log Z=12+\log (\mathrm{O} / \mathrm{H})$ is the metallicity on the Pettini \& Pagel (2004) scale estimated from the mass-metallicity relation

$\log Z=8.74-0.087 \times\left(\log \left(\mathrm{M}_{\star}\right)-b\right)^{2}$,

with $b=10.4+4.46 \times \log (1+z)-1.78 \times(\log (1+z))^{2}($ Genzel et al. 2015 , and references therein). This metallicity correction leads to a mean $\alpha_{\mathrm{CO}}=4.4 \pm 0.3 \mathrm{M}_{\odot} /\left(\mathrm{K} \mathrm{km} \mathrm{s}^{-1} \mathrm{pc}^{2}\right)$ within the sample. Still following the PHIBSS2 methodology, we assume $r_{31}=0.56$ and $r_{41}=0.42$ as suggested by observations in low- and high-redshift SFGs (e.g., Weiss et al. 2007; Dannerbauer et al. 2009; Bothwell et al. 2013; Bolatto et al. 2015; Daddi et al. 2015). From the molecular gas mass upper limits, we derive upper limits for the molecular gas-to-stellar mass ratio $\mu_{\text {gas }}=M_{\text {gas }} / M_{\star}$ and depletion time $t_{\mathrm{depl}}=M_{\mathrm{gas}} / \mathrm{SFR}$. Table 3 indicates the quantities derived from the $\mathrm{CO}$ molecular gas measurements.

Fig. 2 shows how the $\mathrm{CO}(1-0)$ intrinsic luminosity directly derived from the observations (assuming $r_{31}$ and $r_{41}$ as indicated above) correlates with the [O II] SFR and compare with the correlation observed for SFGs within the larger Tacconi et al. (2018) sample and the fitting function proposed by Sargent et al. (2014). The Tacconi et al. (2018) samples includes about $650 \mathrm{CO}$ molecular gas measurements between redshift $z=0$ and 3.4, from which we selected those within 1 dex of the MS line $(0.1<\delta$ MS $<10)$, while the previous Sargent et al. (2014) compilation included 131 of these measurements. Our $3 \sigma L_{\mathrm{CO}(1-0)}$ upper limits and the stacked values with their error bars (cf. Section 3.3) are compatible with the scatter of the relation.

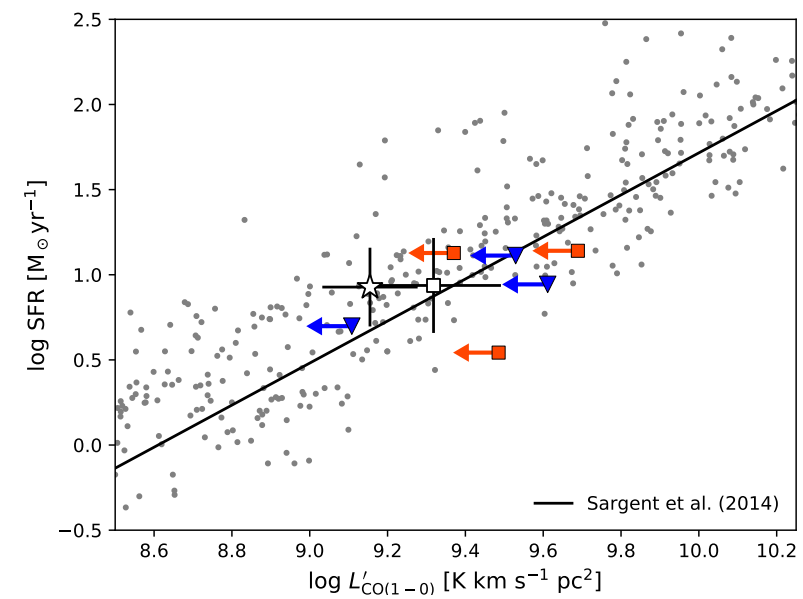

Figure 2. Observed relation between the SFR and the intrinsic CO(1-0) luminosity for the six galaxies of the sample (plain blue triangles and red squares) and the Tacconi et al. (2018) sample of SFGs within 1 dex of the MS line (gray points). Blue triangles correspond to accretion cases, red squares to outflow cases. The empty star with error bars corresponds to the stack of all the sample galaxies; the empty square with error bars to the stack of outflow cases. The solid black line corresponds to the Sargent et al. (2014) fitting function. Our measurements lie within the scatter of the relation.

\subsection{Comparison to existing molecular gas scaling relations}

Figs. 3 and 4 compare the molecular gas-to-stellar mass ratio $\left(\mu_{\text {gas }}=M_{\text {gas }} / M_{\star}\right)$ and depletion time $\left(t_{\text {depl }}=M_{\text {gas }} / \mathrm{SFR}\right)$ upper limits for the six galaxies of the sample to the Tacconi et al. (2018) sample and scaling relations. These scaling relations express $\mu_{\text {gas }}$ and $t_{\mathrm{depl}}$ as a function of redshift $z$, stellar mass $M_{\star}$, and the specific star formation rate $\left(\mathrm{sSFR}=\mathrm{SFR} / M_{\star}\right)$ offset from the MS, $\delta \mathrm{MS}=\operatorname{sSFR} / \operatorname{sSFR}\left(\mathrm{MS} ; z, M_{\star}\right)$, where $\operatorname{sSFR}\left(\mathrm{MS} ; z, M_{\star}\right)$ is the mean sSFR on the MS given $z$ and $M_{\star}$. We use here the Speagle et al. (2014) parametrisation of $\operatorname{sSFR}\left(\mathrm{MS} ; z, M_{\star}\right.$ ). These relations were established on a very large sample of about $1400 \mathrm{CO}$ and dust molecular gas measurements in the range $z=0-4.5$, owing notably to the COLDGASS (Saintonge et al. 2011a,b), PHIBSS (Tacconi et al. 2010, 2013), and PHIBSS2 (Freundlich et al. 2019) programs. They notably quantify how the molecular gas fraction decreases steeply with time while the depletion time increases slightly such that the cosmic evolution of the SFR is mainly driven by the available molecular gas reservoirs. They also show how the gas fraction increases above the MS and decreases below, while the depletion time follows the opposite trend.

Fig. 3 shows the redshift evolution of $\mu_{\text {gas }}$ and $t_{\text {depl }}$ from the Tacconi et al. (2018) sample ${ }^{1}$ together with the upper limits we obtain in the redshift range $z=0.6-1.1$. Fig. 4 further shows $\mu_{\text {gas }} / \mu_{\text {gas }}(\mathrm{MS})$ and $t_{\text {depl }} / t_{\text {depl }}(\mathrm{MS})$ as a function of $\delta \mathrm{MS}$, where $\mu_{\text {gas }}(\mathrm{MS})$ and $t_{\text {depl }}(\mathrm{MS})$ are the molecular gas-to-stellar mass ratio and depletion time on the MS line ( $\delta \mathrm{MS}=1$ ) according to the scaling relations given the redshift and stellar mass of the sources. The expected dependence as a function of $\delta \mathrm{MS}$ and its scatter is shown as the plain and dashed black lines. The upper limits are compatible with the scaling relations, and there is no clear difference between

\footnotetext{
1 We highlight that the scaling relation lines shown in Fig. 3 were scaled to the average stellar mass of the sample and hence are slightly offsetted from the Tacconi et al. (2018) data.
} 

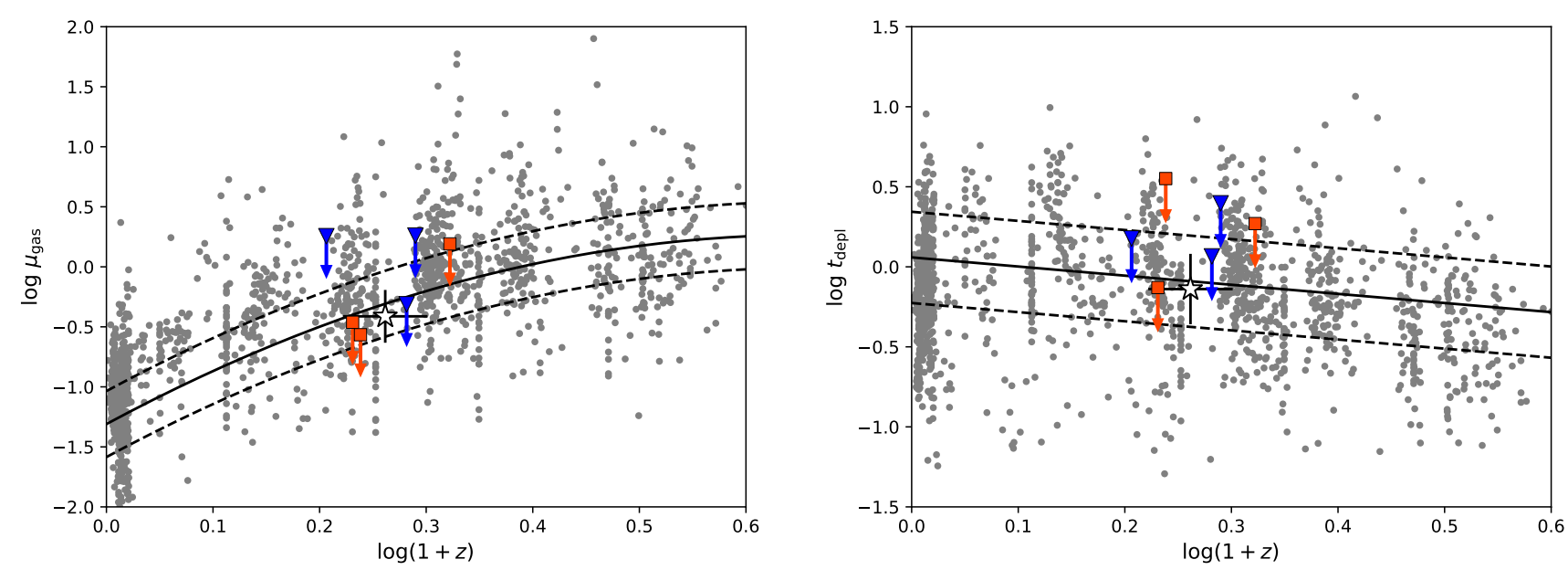

Figure 3. Molecular gas-to-stellar mass ratio and depletion time as a function of redshift for the six galaxies of the sample (plain blue triangles and red squares) compared to the Tacconi et al. (2018) sample (gray points). Blue triangles correspond to accretion cases, red squares to outflow cases. The empty star with error bars corresponds to the stack of all the sample galaxies. The plain black lines correspond to the Tacconi et al. (2018) scaling relations for galaxies of the mean stellar mass of the sample $\left(\log \left(M_{\star} / M_{\odot}\right)=10.3\right)$ on the MS $(\delta \mathrm{MS}=1)$, the dashed lines to their scatter. Since the average mass in each redshift bin of the Tacconi et al. (2018) sample may differ from $\log \left(M_{\star} / M_{\odot}\right)=10.3$, the lines are slightly offsetted compared to the gray points. With an average offset from the MS of -0.07 dex, the stacked detection corresponds to a molecular gas-to-stellar mass ratio slightly below the MS line.
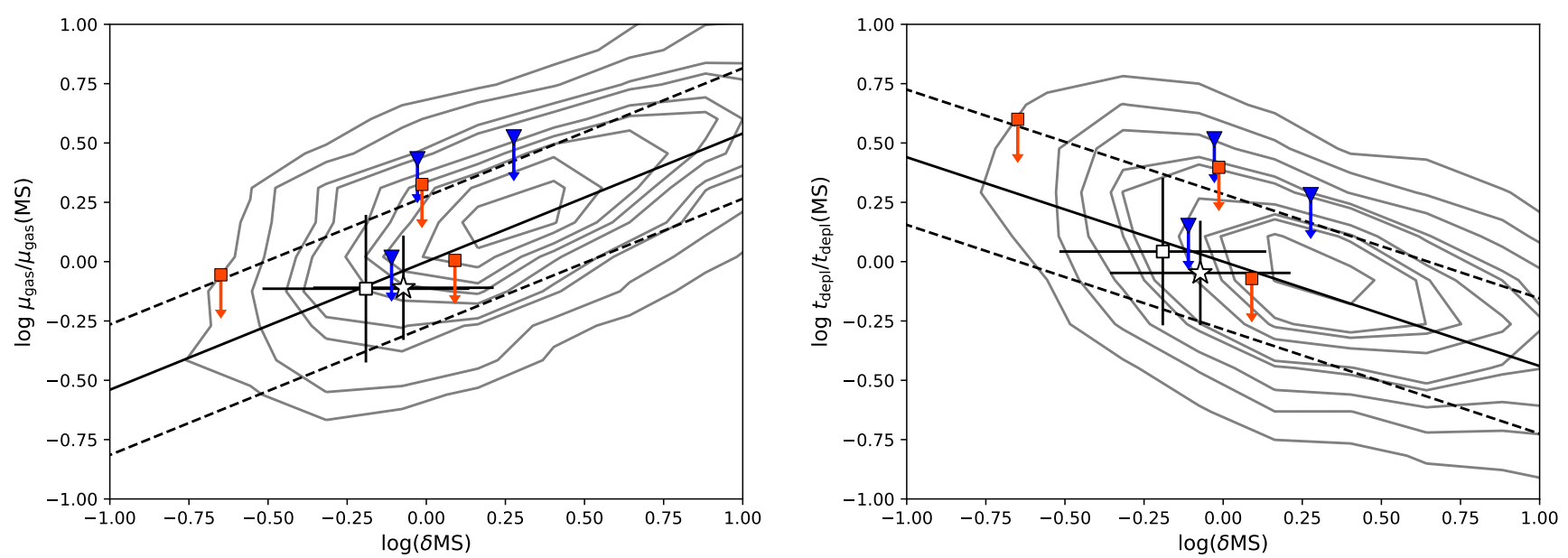

Figure 4. Molecular gas-to-stellar mass ratio and depletion time divided by their average values on the MS given the redshifts and stellar masses of the sources according to the Tacconi et al. (2018) scaling relations as a function of the sSFR offset from the MS, $\delta \mathrm{MS}=\mathrm{sSFR} / \mathrm{sSFR}(\mathrm{MS})$. Blue triangles correspond to accretion cases, red squares to outflow cases. The empty star with error bars corresponds to the stack of all the sample galaxies; the empty square with error bars to the stack of outflow cases. The gray background contours, the black lines, and the dashed lines respectively show the Tacconi et al. (2018) sample, scaling relations, and scatter.

the accretion and outflow subsamples (respectively in blue and red on the figure). More precisely, if we assume a Gaussian scatter in the Tacconi et al. (2018) scaling relations, we can assess the probability for six data points following the corresponding distribution to be simultaneously below the upper limits we obtain, and we find a probability of about $60 \%$.

\subsection{Stacked CO analysis}

We stack the CO spectra at the optical positions of the six observed galaxies, rescaling the $\mathrm{CO}(4-3)$ fluxes to $\mathrm{CO}(3-2)$ fluxes assuming a factor 1.3 between the two line fluxes and normalising the velocities by the MUSE [O II] FWHM. We search for a signal within the FWTM, i.e., within FWHM $\times \sqrt{\log (10) / \log (2)}$, where $97 \%$ of the line flux should be. The resulting stacked spectra for all the galaxies of the sample and for the outflow cases are shown in Fig. 5. In these two cases, we detect velocity-integrated line fluxes respectively of $0.191 \mathrm{Jy} \mathrm{km} / \mathrm{s}$ and $0.271 \mathrm{Jy} \mathrm{km} / \mathrm{s}$ with SNR $=3.2$ and 3.0, noise being evaluated from the standard deviation of the stacked flux over the FWTM. For the accretion cases, the stacked spectrum does not display any detection (the SNR would be $\sim 1.5$ ) but enables to place a $3 \sigma$ upper limit of $0.217 \mathrm{Jy} \mathrm{km} / \mathrm{s}$. The $3 \sigma$ detections are obtained almost without any free parameter since both the central velocities and the line widths are contrained by the MUSE [O II] observations, such that we can be confident in these detections even if the error is significant. We only allow a minor offset in the velocity range where the stacked signal is assessed (the vertical dashed lines in Fig. 5 are not exactly centered on zero). Weighting the different spectra by their RMS noise instead of assuming equal weights lead to stacked integrated $\mathrm{CO}(3-2)$ line fluxes of $0.159 \mathrm{Jy} \mathrm{km} / \mathrm{s}$ 

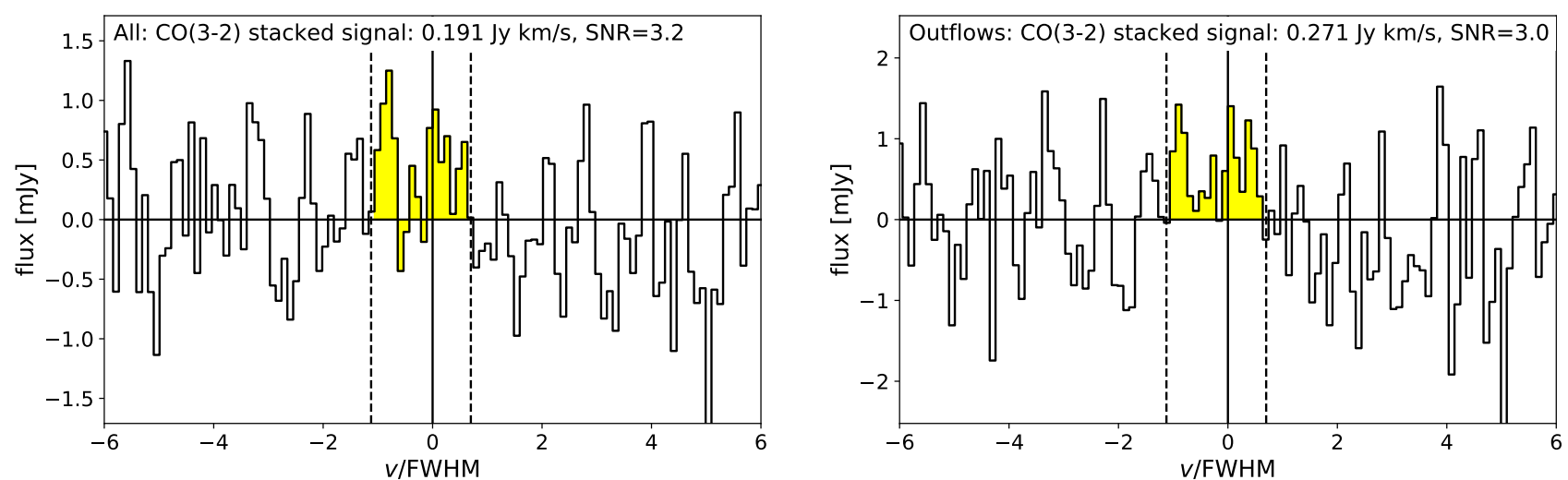

Figure 5. Stacked CO spectra over all galaxies (left) and over the three outflow cases (right). The $\mathrm{CO}(4-3)$ spectra were rescaled to $\mathrm{CO}(3-2)$ assuming a factor $r_{31} / r_{41}=1.3$ between the two line fluxes. The velocity axes were scaled to the FWHM before being stacked and we searched for a signal within the FWTM, i.e., within FWHM $\times \sqrt{\log (10) / \log (2)}$, allowing a minor offset. All spectra were given the same weight. The vertical lines indicate the FWTM, the horizontal ones the flux standard deviation used to determine the SNR. For all galaxies of the sample, we obtain a signal of $0.191 \mathrm{Jy} \mathrm{km} / \mathrm{s}(\mathrm{SNR}=3.2)$, for the outflow cases of $0.271 \mathrm{Jy} \mathrm{km} / \mathrm{s}(\mathrm{SNR}=3.0)$. We did not detect any signal for the stack of the accretion cases, which corresponds to a $0.217 \mathrm{Jy} \mathrm{km} / \mathrm{s} 3 \sigma \mathrm{upper}$ limit.

$(\mathrm{SNR}=2.9)$ for all sources, $0.219 \mathrm{Jy} \mathrm{km} / \mathrm{s}(\mathrm{SNR}=2.8)$ for the outflow cases, and a $0.220 \mathrm{Jy} \mathrm{km} / \mathrm{s}$ upper limit for the accretion cases. Given the SNR, the relative uncertainty on the stacked CO line fluxes is $1 / \mathrm{SNR} \sim 30 \%$, such that the values with and without weighting agree well with one another. Using the $r_{31}$ line ratio and the average redshifts, the stacked CO line fluxes and upper limits correspond respectively to intrinsic $\mathrm{CO}(1-0)$ luminosities equal to $2.0,2.9$, and $2.210^{9} \mathrm{~K} \mathrm{~km} \mathrm{~s}^{-1} \mathrm{pc}^{2}$. We further derive molecular gas masses, gas-to-stellar mass ratios, and depletion times as in Section 3.1, taking the average $\alpha_{\mathrm{CO}}$ conversion factor to estimate the molecular gas mass and the average SFR and stellar mass to estimate the mass ratio and depletion time. The $30 \%$ uncertainty on the CO line fluxes together with the $30 \%$ uncertainty on the Galactic conversion factor (Bolatto et al. 2013), the systematic difference up to $20 \%$ between the metallicity corrections of Bolatto et al. (2013) and Genzel et al. (2012), reflecting the scatter in the $\alpha_{\mathrm{CO}}-$ metallicity relation, and a $20 \%$ uncertainty on the line ratios (e.g., Daddi et al. 2015) leads to a systematic uncertainty of at least $50 \%(0.3 \mathrm{dex})$ on the stacked molecular gas masses.

We include the stacked data points and upper limit in Figs. 2, 3 and 4 . The vertical error bars of the stacked data points assume 0.2 dex uncertainties in SFR and $M_{\star}$ in addition to the 0.3 dex uncertainty in molecular gas mass. The horizontal error bars reflect the $x$-axis spread of the data points. In Fig. 3, we can see that the average molecular gas-to-stellar mass ratio is slighly below its expected MS value given the redshifts and stellar masses of the sample due to the average -0.23 dex $\delta \mathrm{MS}$ offset below the MS, but that the average depletion time is well aligned with the expected MS value. In Fig. 4, we can see that both the stacked values for the whole sample and those for the outflow cases lie on their expected values from the Tacconi et al. (2018) scaling relations. The stacked upper limits for the accretion cases is also compatible with the scaling relations, notably as $65 \%$ of the Gaussian distribution following the scatter in the scaling relations would fall below this upper limit. This indicates (i) that the molecular gas reservoirs of galaxies selected for their strong $\mathrm{Mg}$ II absorption along quasar lineof-sights do not deviate significantly from those of mass-selected samples on average and (ii) that galaxies identified with accreting gas may not have specifically high molecular gas content. Point (i) notably means that the Tacconi et al. (2018) scaling relations can be used to estimate the expected CO fluxes from the MUSE [O II]
SFRs in future molecular gas follow-up studies of MEGAFLOW sources, as was already indicated in Fig. 2. Point (ii) is potentially at odds with expectations from the quasi equilibrium and compaction models, since accretion should replenish the gas reservoirs and enhance star formation. But the error bars of our stacked measurements are large, and mass-selected samples do include accreting and outflowing galaxies such that their molecular gas properties may already reflect the different configurations. This trend needs to be confirmed by individual detections and larger samples, for example using the Atacama Large Millimeter/Submillimiter Array (ALMA).

\subsection{Dust continuum}

In addition to $\mathrm{CO}$, the full bandwidth of the NOEMA observations enables to assess the dust continuum fluxes, indicated with their RMS errors in Table 4. We report no individual detection, but these estimates enable to place individual $3 \sigma$ upper limits and can also be combined to provide a stacked result of $0.4 \pm 24 \mu \mathrm{Jy}$ as well as a $3 \sigma$ stacked upper limit of $73 \mu \mathrm{Jy}$ at an effective frequency of 220 $\mathrm{GHz}$, weighting each value by the RMS error.

Long-wavelength dust emission can be used to probe the interstellar medium mass since the Rayleigh-Jeans tail of this emission is almost always optically thin (e.g., Scoville et al. 2014, 2016) and hence to provide an additional measurement of the gas mass. We estimate upper upper limits for the individual and stacked molecular gas masses from the dust continuum emission according to the Scoville et al. (2016) calibration, assuming a dust temperature $T_{d}=25 \mathrm{~K}$ and an emissivity power-law index $\beta=1.8$. Namely, the molecular gas mass derived from the dust continuum is expressed as

$M_{\text {gas,dust }}=\frac{s_{\text {dust }} D_{L}^{2}}{\kappa\left(v_{\text {rest }}\right) 2 k T_{d}\left(v_{\text {rest }} / c\right)^{2} \Gamma_{\mathrm{RJ}}\left(T_{d}, v_{\text {obs }}, z\right)(1+z)}$

where $s_{\text {dust }}$ is the observed flux density, $D_{L}$ the luminosity distance, $\kappa(v)=\kappa\left(v_{850 \mu \mathrm{m}}\right)(\lambda / 850 \mu \mathrm{m})^{-\beta}$ the dust opacity, with $\kappa\left(v_{850 \mu \mathrm{m}}\right)=4.8410^{-4} \mathrm{~m}^{2} \mathrm{~kg}^{-1}$, and $\Gamma_{\mathrm{RJ}}\left(T_{d}, v_{\mathrm{obs}}, z\right)=h v_{\mathrm{obs}}(1+$ $z) / k T_{d} /\left(\exp \left(h v_{\mathrm{obs}}(1+z) / k T_{d}\right)-1\right)$ the correction for departure in the rest-frame of the Planck function from Rayleigh-Jeans. The dust continuum emission can also translate into an infrared (IR) luminosity or equivalently in an IR SFR, $\mathrm{SFR}_{\mathrm{IR}}$, using the Magdis et al. (2012) templates with the mean redshift of the sample $(z=$ 
Table 4. Dust continuum measurements: RMS error, effective frequency, ISM mass, and IR SFR. The table includes the individual $3 \sigma$ upper limits and the stacked values for all galaxies, for the accretion cases (A), and for the wind cases $(\mathrm{W})$. We stress that the ISM mass and the IR SFR are not independent since they are both derived from the continuum flux. Galaxies are referred to with their short ID (cf. Table 1).

\begin{tabular}{lcccc}
\hline \hline ID & $\begin{array}{c}\sigma_{\text {cont }} \\
{[\mu \mathrm{Jy}]}\end{array}$ & $\begin{array}{c}v_{\text {cont }} \\
{[\mathrm{GHz}]}\end{array}$ & $\begin{array}{c}M_{\text {gas,dust }} \\
{\left[M_{\odot}\right]}\end{array}$ & $\begin{array}{c}\mathrm{SFR}_{\mathrm{IR}} \\
{\left[\mathrm{M}_{\odot} \mathrm{yr}^{-1}\right]}\end{array}$ \\
\hline 0608 & 55 & 209.1 & $<1.710^{10}$ & $<24.3$ \\
0912 & 51 & 234.8 & $<1.210^{10}$ & $<39.5$ \\
0949 & 68 & 230.3 & $<1.710^{10}$ & $<56.6$ \\
\hline 1099 & 75 & 213.5 & $<2.510^{10}$ & $<72.0$ \\
0702 & 45 & 209.0 & $<1.510^{10}$ & $<23.8$ \\
0731 & 107 & 260.8 & $<1.510^{10}$ & $<58.8$ \\
\hline stack & 24 & 220.8 & $<6.910^{9}$ & $<15.2$ \\
A stack & 33 & 224.6 & $<8.710^{9}$ & $<20.7$ \\
W stack & 36 & 216.1 & $<1.110^{10}$ & $<22.3$ \\
\hline
\end{tabular}

0.83), the typical temperature for MS galaxies at this epoch and a Chabrier (2003) IMF. We derive individual and stacked upper limits for these quantities, namely $M_{\text {gas,dust }}<6.910^{9} M_{\odot}$ and $\mathrm{SFR}_{\mathrm{IR}}<15.2 M_{\odot} \mathrm{yr}^{-1}$ for the stack over all galaxies. Table 4 indicates the quantities derived from the dust continuum upper limits. We stress that a given observation can not simultaneously constrain $M_{\text {gas,dust }}$ and $\mathrm{SFR}_{\mathrm{IR}}$ since these quantities are not independent from each other here.

The dust continuum molecular gas mass upper limits are consistent with the $\mathrm{CO}$ molecular gas upper limits indicated in Table 3 and fall within 0.3 dex of one another, which is the typical uncertainty of such measurements. The [O $\left.{ }_{\text {II }}\right]$ SFR fall within the IR SFR upper limits. In particular, the $\mathrm{SFR}_{\mathrm{IR}}$ stacked upper limit $\left(<15.2 \mathrm{M}_{\odot} \mathrm{yr}^{-1}\right)$ fits well with the average [O II] SFR $\left(9.6 M_{\odot} \mathrm{yr}^{-1}\right)$.

\section{CONCLUSION}

In this paper, we present the NOEMA MEGAFLOW pilot program, a first attempt at probing the molecular gas reservoirs of starforming galaxies (SFGs) whose circum-galactic medium has been observed in absorption along quasar lines-of-sight and which thus have estimates of their mass accretion or ouflow rates. The motivation for such a program is to test the quasi equilibrium ('bathtub') and compaction models describing the evolution of star-forming galaxies along the main sequence (MS) of star formation, with simultaneous measurements of gas flows and gas content. We observe the $\mathrm{CO}(3-2)$ or $\mathrm{CO}(4-3)$ carbon monoxide line and the dust continuum of six galaxies at $z=0.6-1.1$ drawn from the MusE GAs FLOw and Wind (MEGAFLOW) survey (Schroetter et al. 2016, 2019; Zabl et al. 2019, 2020) with the IRAM NOEMA interferometer. Three of these galaxies were identified with accretion around the disc plane, three with outflows perpendicular to the disc (Table 1 and Fig. 1). We report $\mathrm{CO}$ and dust continuum upper limits for the individual galaxies (Tables 3 and 4), and $\mathrm{CO}$ stacked detections over the whole sample and over the outflow cases (Fig. 5). From the $\mathrm{CO}$ data, we derive molecular gas masses, gas-to-stellar mass ratios and depletion times. From the dust continuum data, we derive upper limits for another estimate of the molecular gas content and the IR star formation rate. The dust continuum upper limits are compatible with the $\mathrm{CO}$ molecular gas mass upper limits and the SFR obtained from MUSE [O II] observations. We further de- tect the dust continuum in three of the quasars and a strong line in one of them, which we identify with $\mathrm{CO}$ (4-3) (Appendix B).

Both the upper limits and the stacked results are compatible with the Tacconi et al. (2018) scaling relations obtained from a large sample of mass-selected SFGs on and around the MS (Figs. 2, 3 and 4). This indicates that the molecular gas reservoirs of galaxies selected through their Mg II absorption as the MEGAFLOW galaxies do not deviate significantly from mass-selected samples, such that the scaling relations can be used to estimate the expected $\mathrm{CO}$ fluxes from the Muse [O II] SFRs in future studies. Galaxies identified with accretion may not have specifically high molecular gas content. This may be in tension with the theoretical expectations of the quasi equilibrium and compaction models, since accretion is associated with gas replenishment, but the low signal-to-noise ratios (SNR $=3.2$ and 3.0) of the stacked detections induce relatively large error bars.

This pilot program establishes the feasability of molecular gas studies in galaxies with gas flows, such as those of the MEGAFLOW sample, and shows that molecular gas properties in absorption-selected samples may not fundamentally differ from those of mass-selected samples on average. This not only calls for additional observations to confirm or infirm the result of the stacked detections, but also provides tools to assess the observing time in future proposals, using the Tacconi et al. (2018) scaling relations. The next step consists in individual molecular gas detections in a statistically meaningful sample of galaxies with gas flows in order to quantify how the molecular gas fraction and depletion time depend on the inflow/outflow rates and test whether the gas content follows the expectation from the quasi equilibrium model and the compaction scenario. The current pilot program shows that while limited samples can be observed with NOEMA, ALMA is necessary to significantly increase the sample size and the sensitivity.

\section{ACKNOWLEDGEMENTS}

J. F. would like to thank F. Combes, P. Salomé, and A. Dekel for their valuable insights and advice, $C$. Lefèvre for the guidance during the data reduction, D. Maoz for his support. We thank the anonymous referee for his/her careful reading, which contributed to improve this article. This work is based on observations carried out under project number W18CS with the IRAM NOEMA Interferometer. IRAM is supported by INSU/CNRS (France), MPG (Germany) and IGN (Spain). This work was supported by the ANR 3DGasFlows (ANR-17-CE31-0017) and the OCEVU Labex (ANR-11-LABX-0060). It has received funding from the European Research Council (ERC) under the European Union's FP7 Programme, Grant No. 833031, and was partly supported by the grants France-Israel PICS, I-CORE Program of the PBC/ISF 1829/12, BSF 2014-273, NSF AST-1405962, GIF I-1341-303.7/2016, and DIP STE1869/2-1 GE625/17-1.

\section{DATA AVAILABILITY}

The data underlying this article will be shared on reasonable request to the corresponding author.

\section{REFERENCES}

Alam S., et al., 2015, ApJS, 219, 12

Alcorn L. Y., et al., 2018, ApJ, 858, 47 
Bacon R., et al., 2006, The Messenger, 124, 5

Bacon R., et al., 2010, in Proc. SPIE. p. 773508, doi:10.1117/12.856027

Birnboim Y., Dekel A., 2003, MNRAS, 345, 349

Bolatto A. D., Wolfire M., Leroy A. K., 2013, ARA\&A, 51, 207

Bolatto A. D., et al., 2015, ApJ, 809, 175

Boogaard L. A., et al., 2018, A\&A, 619, A27

Bordoloi R., et al., 2011, ApJ, 743, 10

Bothwell M. S., et al., 2013, MNRAS, 429, 3047

Bouché N., et al., 2010, ApJ, 718, 1001

Bouché N., Hohensee W., Vargas R., Kacprzak G. G., Martin C. L., Cooke J., Churchill C. W., 2012, MNRAS, 426, 801

Bouché N., Murphy M. T., Kacprzak G. G., Péroux C., Contini T., Martin C. L., Dessauges-Zavadsky M., 2013, Science, 341, 50

Bouché N., Carfantan H., Schroetter I., Michel-Dansac L., Contini T., 2015, AJ, 150, 92

Bouché N., et al., 2016, ApJ, 820, 121

Calzetti D., Armus L., Bohlin R. C., Kinney A. L., Koornneef J., StorchiBergmann T., 2000, ApJ, 533, 682

Caputi K. I., et al., 2006, ApJ, 637, 727

Chabrier G., 2003, PASP, 115, 763

Chen H.-W., Wild V., Tinker J. L., Gauthier J.-R., Helsby J. E., Shectman S. A., Thompson I. B., 2010, ApJ, 724, L176

Conroy C., Wechsler R. H., 2009, ApJ, 696, 620

Daddi E., et al., 2005, ApJ, 626, 680

Daddi E., et al., 2007, ApJ, 670, 156

Daddi E., et al., 2010, ApJ, 713, 686

Daddi E., et al., 2015, A\&A, 577, A46

Dannerbauer H., Daddi E., Riechers D. A., Walter F., Carilli C. L., Dickinson M., Elbaz D., Morrison G. E., 2009, ApJ, 698, L178

Davé R., Oppenheimer B. D., Finlator K., 2011, MNRAS, 415, 11

Davé R., Finlator K., Oppenheimer B. D., 2012, MNRAS, 421, 98

Dekel A., Birnboim Y., 2006, MNRAS, 368, 2

Dekel A., Burkert A., 2014, MNRAS, 438, 1870

Dekel A., et al., 2009, Nature, 457, 451

Dekel A., Zolotov A., Tweed D., Cacciato M., Ceverino D., Primack J. R., 2013, MNRAS, 435, 999

Dekel A., Lapiner S., Dubois Y., 2019, arXiv e-prints, p. arXiv:1904.08431

Dekel A., et al., 2020a, arXiv e-prints, p. arXiv:2003.08984

Dekel A., Ginzburg O., Jiang F., Freundlich J., Lapiner S., Ceverino D., Primack J., 2020b, MNRAS, 493, 4126

Dekker H., D’Odorico S., Kaufer A., Delabre B., Kotzlowski H., 2000, in Iye M., Moorwood A. F., eds, Society of Photo-Optical Instrumentation Engineers (SPIE) Conference Series Vol. 4008, Proc. SPIE. pp 534545, doi: $10.1117 / 12.395512$

Elbaz D., et al., 2007, A\&A, 468, 33

Elbaz D., et al., 2011, A\&A, 533, A119

Erb D. K., Steidel C. C., Shapley A. E., Pettini M., Reddy N. A., Adelberger K. L., 2006, ApJ, 646, 107

Feldmann R., 2013, MNRAS, 433, 1910

Förster Schreiber N. M., et al., 2006, ApJ, 645, 1062

Freundlich J., et al., 2013, A\&A, 553, A130

Freundlich J., et al., 2019, A\&A, 622, A105

Garn T., Best P. N., 2010, MNRAS, 409, 421

Genel S., Bouché N., Naab T., Sternberg A., Genzel R., 2010, ApJ, 719, 229

Genzel R., et al., 2006, Nature, 442, 786

Genzel R., et al., 2008, ApJ, 687, 59

Genzel R., et al., 2012, ApJ, 746, 69

Genzel R., et al., 2013, ApJ, 773, 68

Genzel R., et al., 2015, ApJ, 800, 20

Kacprzak G. G., Churchill C. W., Evans J. L., Murphy M. T., Steidel C. C., 2011, MNRAS, 416, 3118

Kacprzak G. G., Churchill C. W., Nielsen N. M., 2012, ApJ, 760, L7

Kacprzak G. G., et al., 2014, ApJ, 792, L12

Kereš D., Katz N., Weinberg D. H., Davé R., 2005, MNRAS, 363, 2

Kereš D., Katz N., Fardal M., Davé R., Weinberg D. H., 2009, MNRAS, 395,160

Kewley L. J., Geller M. J., Jansen R. A., 2004, AJ, 127, 2002
Lanzetta K. M., Bowen D., 1990, ApJ, 357, 321

Lilly S. J., Carollo C. M., Pipino A., Renzini A., Peng Y., 2013, ApJ, 772, 119

Magdis G. E., et al., 2012, ApJ, 760, 6

Masci F., 2011, http://wise2.ipac.caltech.edu/staff/fmasci/ UpperLimits_FM2011.pdf

Muzahid S., Kacprzak G. G., Churchill C. W., Charlton J. C., Nielsen N. M., Mathes N. L., Trujillo-Gomez S., 2015, ApJ, 811, 132

Nielsen N. M., Churchill C. W., Kacprzak G. G., 2013, ApJ, 776, 115

Noeske K. G., et al., 2007, ApJ, 660, L43

Ocvirk P., Pichon C., Teyssier R., 2008, MNRAS, 390, 1326

Peng Y.-j., Maiolino R., 2014, MNRAS, 443, 3643

Peng Y.-j., et al., 2010, ApJ, 721, 193

Péroux C., Nelson D., van de Voort F., Pillepich A., Marinacci F., Vogelsberger M., Hernquist L., 2020, MNRAS, 499, 2462

Pettini M., Pagel B. E. J., 2004, MNRAS, 348, L59

Renzini A., Peng Y.-j., 2015, ApJ, 801, L29

Rodighiero G., et al., 2010, A\&A, 518, L25

Roediger J. C., Courteau S., 2015, MNRAS, 452, 3209

Ross N. P., et al., 2012, ApJS, 199, 3

Saintonge A., et al., 2011a, MNRAS, 415, 32

Saintonge A., et al., 2011b, MNRAS, 415, 61

Sargent M. T., Béthermin M., Daddi E., Elbaz D., 2012, ApJ, 747, L31

Sargent M. T., et al., 2014, ApJ, 793, 19

Schiminovich D., et al., 2007, ApJS, 173, 315

Schroetter I., Bouché N., Péroux C., Murphy M. T., Contini T., Finley H., 2015, ApJ, 804, 83

Schroetter I., et al., 2016, ApJ, 833, 39

Schroetter I., et al., 2019, MNRAS, 490, 4368

Schuster K.-F., 2014, IRAM Annual Reports, http://www. iram-institute.org/medias/uploads/IRAM_2014.pdf

Scoville N., et al., 2014, ApJ, 783, 84

Scoville N., et al., 2016, ApJ, 820, 83

Seko A., Ohta K., Yabe K., Hatsukade B., Akiyama M., Tamura N., Iwamuro F., Dalton G., 2016, ApJ, 833, 53

Solomon P. M., Downes D., Radford S. J. E., Barrett J. W., 1997, ApJ, 478, 144

Speagle J. S., Steinhardt C. L., Capak P. L., Silverman J. D., 2014, ApJS, 214,15

Stark D. P., Swinbank A. M., Ellis R. S., Dye S., Smail I. R., Richard J., 2008, Nature, 455, 775

Steidel C. C., 1995, in Meylan G., ed., QSO Absorption Lines. p. 139 (arXiv: astro-ph/9509098)

Tacchella S., Dekel A., Carollo C. M., Ceverino D., DeGraf C., Lapiner S., Mandelker N., Primack J. R., 2016a, MNRAS, 457, 2790

Tacchella S., Dekel A., Carollo C. M., Ceverino D., DeGraf C., Lapiner S., Mandelker N., Primack J. R., 2016b, MNRAS, 458, 242

Tacconi L. J., et al., 2010, Nature, 463, 781

Tacconi L. J., et al., 2013, ApJ, 768, 74

Tacconi L. J., et al., 2018, ApJ, 853, 179

Weiss A., Downes D., Walter F., Henkel C., 2007, in Baker A. J., Glenn J., Harris A. I., Mangum J. G., Yun M. S., eds, Astronomical Society of the Pacific Conference Series Vol. 375, From Z-Machines to ALMA: (Sub)Millimeter Spectroscopy of Galaxies. p. 25

Wendt M., Bouché N. F., Zabl J., Schroetter I., Muzahid S., 2020, arXiv e-prints, p. arXiv:2009.08464

Werk J. K., Prochaska J. X., Thom C., Tumlinson J., Tripp T. M., O’Meara J. M., Peeples M. S., 2013, ApJS, 204, 17

Whitaker K. E., van Dokkum P. G., Brammer G., Franx M., 2012, ApJ, 754, L29

Whitaker K. E., et al., 2014, ApJ, 795, 104

Wuyts S., et al., 2011a, ApJ, 738, 106

Wuyts S., et al., 2011b, ApJ, 742, 96

Zabl J., et al., 2019, MNRAS, 485, 1961

Zabl J., et al., 2020, MNRAS, 492, 4576

Zhu G., Ménard B., 2013, ApJ, 770, 130

Zolotov A., et al., 2015, MNRAS, 450, 2327

van der Wel A., et al., 2014, ApJ, 788, 28 


\section{J. Freundlich et al.}

\section{APPENDIX A: CO SPECTRA}

We display in Fig. A1 the CO spectra for the six galaxies of the sample observed with NOEMA.

\section{APPENDIX B: QUASAR DETECTIONS}

Although our main targets are the galaxies observed in MgII absorption, we clearly detect the dust continuum emission of quasars J0800p1849 and J0937p0656 and tentatively that of J1039p0714. Upper limits can be determined from the RMS noise for the other three quasars. Table B1 summarizes these continuum observations, while Fig. B1 shows the continuum maps associated to the detected quasars. Quasar J0800p1849 is spatially resolved with a FWHM of $1.65 \pm 0.22 \operatorname{arcsec}(14 \mathrm{kpc}$ at the quasar redshift, $z=1.294)$, which may indicate a large host galaxy.

We further extract the spectrum at the optical position of each quasar by fitting the PSF in the $u v$ space with go uvfit. Although most of these spectra do not display any specific feature, we observe a bright $\mathrm{SNR}=7.3$ emission line at $v_{\mathrm{obs}}=200.466 \mathrm{GHz}$ for J0800p1849. The spectrum is shown in Fig. B2, and we fit the line with a Gaussian to assess its integrated flux. This line may be associated with $\mathrm{CO}(4-3)\left(v_{\text {rest }}=461.041 \mathrm{GHz}\right)$ at the quasar redshift $(z=1.294)$, albeit with a $\sim 700 \mathrm{~km} / \mathrm{s}$ velocity offset which could be due to broad features, wings, or uncertainties in the quasar redshift. This association is reinforced by the presence of a line that could correspond to $[\mathrm{O} \mathrm{II}]$ with a comparable offset in the MUSE data. Assuming a Galactic conversion factor and a $\mathrm{CO}(4-3) / \mathrm{CO}(1-0)$ ratio of 0.42 , the integrated line flux yields a molecular gas mass of $2.210^{11} M_{\odot}$. Alternatively, the line could also correspond to the emission of an otherwise undetected galaxy on the line of sight, for example $\mathrm{CO}(2-1)\left(v_{\text {rest }}=230.538 \mathrm{GHz}\right)$ at $z=0.150$, since none of the detected $\mathrm{Mg}$ II absorbers on the line-of-sight of this quasar is expected to display strong emission lines around $v_{\mathrm{obs}}=200.466 \mathrm{GHz}$.

This paper has been typeset from a $\mathrm{T}_{\mathrm{E}} \mathrm{X} / \mathrm{L} \mathrm{T}_{\mathrm{E}} \mathrm{X}$ file prepared by the author. 

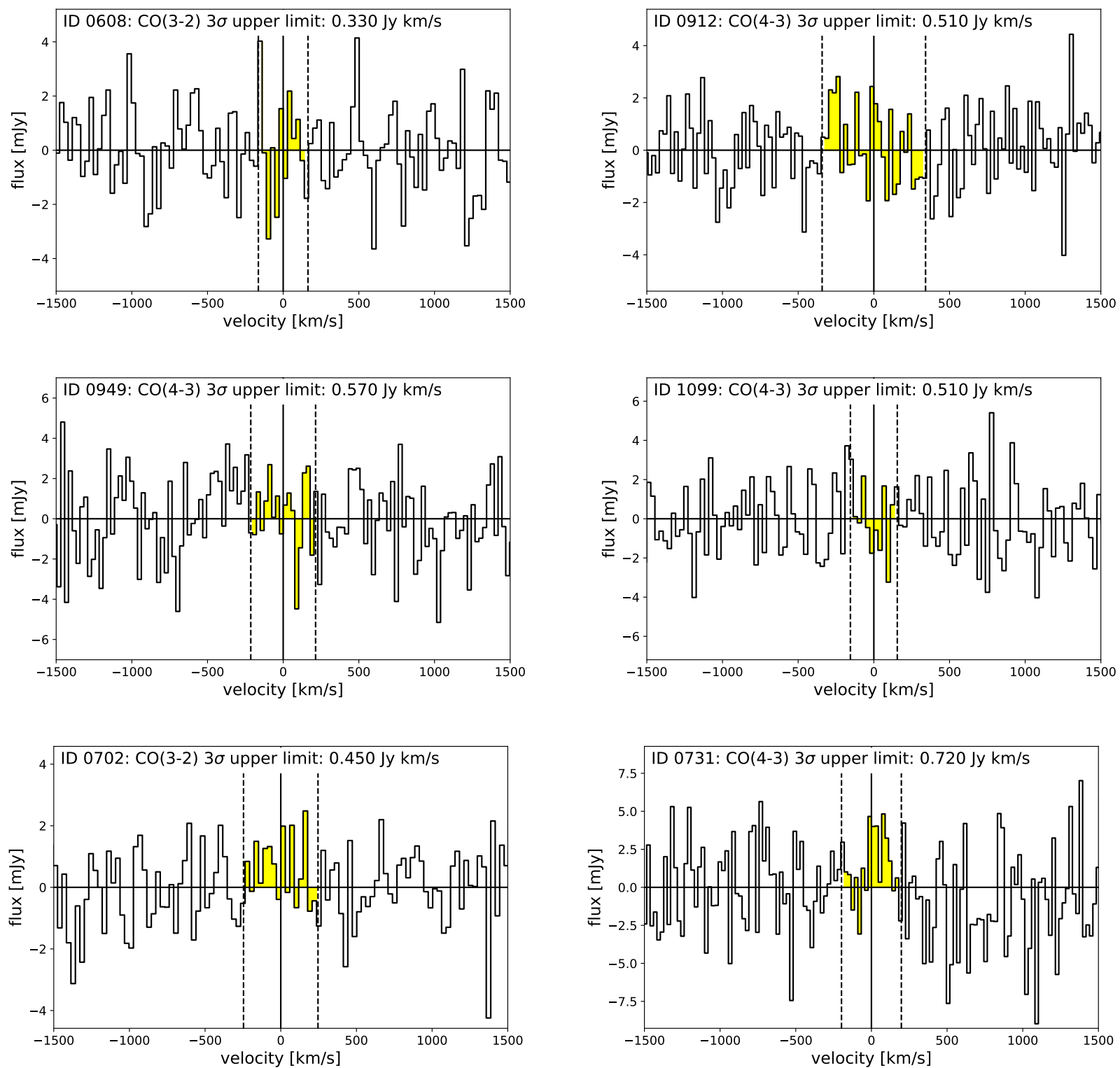

Figure A1. CO spectra of the six galaxies of the NOEMA MEGAFLOW sample extracted at the optical position, fitting the PSF in $u v$ space. The plain vertical line corresponds to $v=0 \mathrm{~km} / \mathrm{s}$, the two vertical dashed lines highlight the FWTM derived from the MUSE [OII] FWHM assuming a Gaussian line. We calculate the signal within the FWTM but do not find any detection with SNR $\geq 3$. Galaxies 0702 and 0731 may display hints of detection at low SNR, with integrated line fluxes within the FWTM respectively equal to $0.280 \mathrm{Jy} \mathrm{km} / \mathrm{s}(\mathrm{SNR}=1.9)$ and $0.567 \mathrm{Jy} \mathrm{km} / \mathrm{s}(\mathrm{SNR}=2.4)$. Galaxies are referred to with their short ID (cf. Table 1).

Table B1. Quasar continuum observations: ID, coordinates and redshift, continuum effective frequency, RMS noise, detected flux or $3 \sigma$ upper limit, and SNR.

\begin{tabular}{llllllll}
\hline \hline Quasar ID & R.A. & DEC. & $z$ & $v_{\text {cont }}[\mathrm{GHz}]$ & $\sigma_{\text {cont }}[\mathrm{mJy}]$ & $s_{\text {cont }}[\mathrm{mJy}]$ & $\mathrm{SNR}$ \\
\hline J0800p1849 & $08: 00: 04.55265$ & $+18: 49: 35.0828$ & 1.294 & 209.1 & 0.107 & 0.485 & $4.5^{\star}$ \\
J1236p0725 & $12: 36: 24.3931$ & $+07: 25: 51.5496$ & 1.606 & 234.9 & 0.051 & $<0.153$ & - \\
J1039p0714 & $10: 39: 36.66840$ & $+07: 14: 27.36$ & 1.536 & 230.3 & 0.077 & 0.179 & 2.3 \\
J0838p0257 & $08: 38: 52.05480$ & $+02: 57: 03.657$ & 1.771 & 213.7 & 0.088 & $<0.263$ & - \\
J0937p0656 & $09: 37: 49.5866$ & $+06: 56: 56.2704$ & 1.802 & 208.6 & 0.053 & 0.354 & 6.7 \\
J0015m0751 & $00: 15: 35.17573$ & $-07: 51: 03.07357$ & 0.874 & 260.4 & 0.111 & $<0.334$ & - \\
\hline
\end{tabular}

Notes. We use the optical position for the quasar, except for J0800p1849 and J0937p065 where the signal is strong enough to release this prior. Typical astrometric corrections are at the level of 0.1-0.2 arcsec. ${ }^{\star}$ The SNR associated to J0800p1849 is artificially low compared to the other two because of the increased number of degrees of freedom enabled by its spatial resolution: the source is indeed resolved (cf. Fig. B1), so we fit it with a Gaussian rather than a point source. The FWHM of the Gaussian is found to be $1.65 \pm 0.22 \mathrm{arcsec}$, i.e., about $14 \mathrm{kpc}$ at the quasar redshift. 

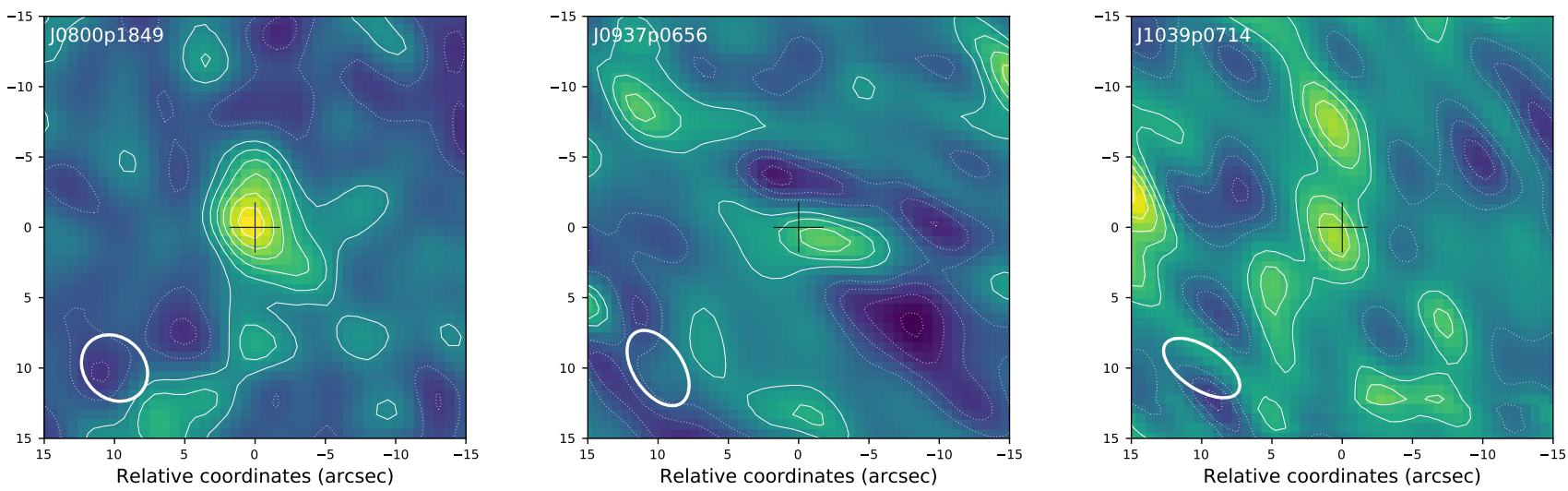

Figure B1. Continuum maps of the detected quasars J0800p1849, J0937p0656, and J1039p0714. The effective frequencies are 209.1, 208.6, and 230.3 GHz. The central cross highlights the quasar location from the Zhu \& Ménard (2013) catalog. Contours correspond to integer $\sigma$ levels (dotted for the negative ones). The white ellipse at the bottom left indicates the beam.

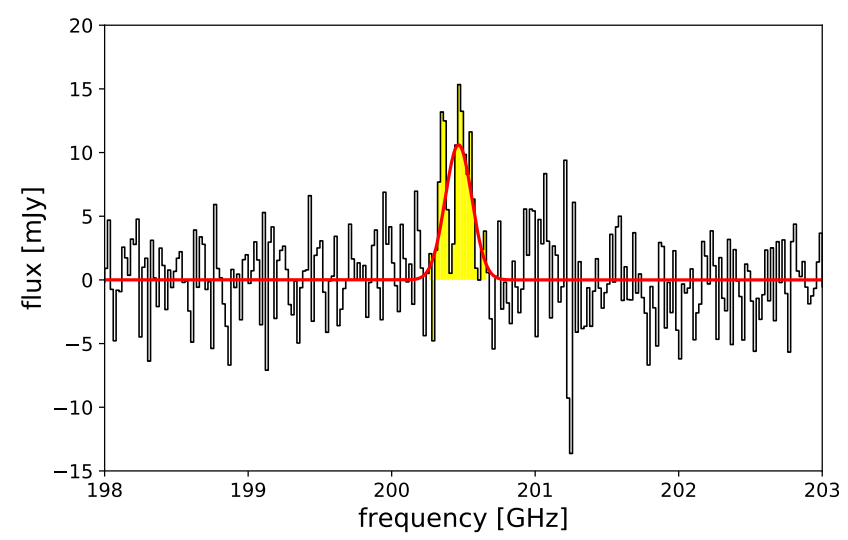

Figure B2. Spectrum of quasar J0800p1849, displaying a bright emission line. We carried out a Gaussian fit to the line using gildas class, yielding $v_{\mathrm{obs}}=200.47 \pm 0.02 \mathrm{GHz}$, a line width equal to $326 \pm 48 \mathrm{~km} / \mathrm{s}$, and an integrated line flux $3.7 \pm 0.5 \mathrm{Jy} \mathrm{km} / \mathrm{s}$. 